\title{
Iterative 3D Point-Set Registration Based on Hierarchical Vertex Signature (HVS)
}

\author{
Jun Feng ${ }^{1}$ and Horace H.S. Ip ${ }^{1,2}$ \\ ${ }^{1}$ Image Computing Group, Department of Computer Science, \\ City University of Hong Kong, Tat Chee Avenue, Kowloon, Hong Kong \\ feng@cs.cityu.edu.hk \\ http://icg.cityu.edu.hk/ICGers/Judy/judy.htm \\ ${ }^{2}$ Centre for Innovative Applications of Internet And Multimedia Technologies (AIMtech), \\ City University of Hong Kong, Tat Chee Avenue, Kowloon, Hong Kong \\ cship@cityu.edu.hk \\ http://icg.cityu.edu.hk/ICGers/hsip/hsip.htm
}

\begin{abstract}
Robust 3D point registration is difficult for biomedical surfaces, especially for roundish and approximate symmetric soft tissues such as liver, stomach, etc. We present an Iterative Optimization Registration scheme (IOR) based on Hierarchical Vertex Signatures (HVS) between point-sets of medical surfaces. HVSs are distributions of concatenated neighborhood angles relative to the PCA axes of the surfaces which concisely describe global structures and local contexts around vertices in a hierarchical paradigm. The correspondences between point-sets are then established by Chi-Square test statistics. Specifically, to alleviate the sensitivity to axes directions that often affects robustness for other global axes based algorithms, IOR aligns surfaces gradually, and incrementally calibrates the directions of major axes in a multi-resolution manner. The experimental results demonstrate IOR is efficient and robust for liver registration. This method is also promising to other applications such as morphological pathological analysis, 3D model retrieval and object recognition.
\end{abstract}

\section{Introduction}

Automatic and robust registration between $3 \mathrm{D}$ images is very important for medical image analysis. Three main purposes of this kind of registrations are: (1) Compare two organ shapes of the same patient in different periods for diagnosis. (2) Register tissue across individuals for physiological analysis. (3) Match 3D data of patients with anatomical atlas for shape representation [1]. The possible primitives of 3D shape registration include points, lines, curves, facets and surfaces [2]. Usually, point registration is also a key step to construct statistical deformable models [3].

The majority of the previous work dealing with 3D point-set registration came from the computer vision community. Most of them were based on shape matching. For example, a well-known method called "Iterative Closest Point" was proposed by Besl and Mckay [4]. It provides a general solution for registration by minimizing the distances between the nearest neighbors in an iterative procedure. However, the process is computationally expensive. Cyr and Kamal generated a number of typical sample $2 \mathrm{D}$ views from the 3D models and matched them against a given view [5]. 
Sundar et al. encoded the topological information of 3D shapes in the form of a skeletal graph and matched them for registration [6]. Modal matching technique performs point registration between objects by their eigenmodes [7,8]. It provides a global to local description of shape deformation; however, the technique requires very expensive calculation for eigenmodes.

Another common approach for point-set registration is to match distinctive local features between surface points such as geometric invariants [9]; these often fail because of insufficient local information and different viewpoint that radically alter local feature appearance. Some authors resorted to stochastic approaches to let the registration process be immune to noise and small deformations. Belongie and Malik [10] introduced a 2D "shape context" which described the coarse distribution of shape with respect to a given point. This descriptor offers a globally discriminative characterization. Yamany et al. [11] presented a "surface signature" to capture the surface curvature information seen from certain points. Chin [12] proposed a "point signature" to describe the structural neighborhood of a point on the 3D surface. Statistics of the distance and relative angles are collected to characterize feature points.

However, these distributions cannot capture the location of the features and they are not always able to provide sufficiently distinctive information to achieve one-toone point registration. This problem is more severe in medical/biomedical image analysis. Usually, shapes of biomedical tissues are very different from those of the man-made objects such as chairs, buildings, cars and aircrafts, etc., investigated in most computer vision applications. There exist large classes of nature objects in biomedical applications that are smooth, rounded, curved, and sometimes approximately symmetric such as livers, heart lenticels, stomachs and kidneys. Vertices on these kinds of organ surfaces frequently share similar geometric features, which often makes local geometric based matching fail.

In this paper, we propose an idea prompted by [11] and [12] to register point-sets defined in segmented biomedical sense data, especially for blobby and approximate symmetric soft tissues. The shapes of these tissues often differ from each other through some form of non-linear deformations. The challenge posed by such models is that it is frequently difficult to define landmarks or to obtain salient geometric features from the surfaces. We present an intuitively simple but very effective and robust feature descriptor called "Hierarchical Vertex Signature" (HVS) for describing the distribution of shape structures in the context of a surface point. Our proposed descriptor captures both global and local structures of the 3D surface from the point of view of any vertex by associating each vertex with a probability distribution of angles between vectors linking pairs of vertices and the major axes of the organs. Registration is then carried out based on the similarity between vertices on different surfaces. Our approach is different from [11] and [12] in: (1)We calculate angles relative to global axes (PCA axes) while they estimate angle values relative to local surface normals. Therefore, our method is more robust to noise and more insensitive to surface smoothness. More importantly, the extension of the relative angles $(R A s)$ defined in a global canonical frame makes HSV has the ability to indicate orientation of a point relative to the whole surface. (2) We construct 1D signature for each point while both of them employ 2D signature feature images so our method is more efficient and 
the similarity comparison is more intuitive and accurate. (3) Our proposed signature is hierarchically defined in different neighborhood field of the vertex; therefore, it provides both local and global shape descriptions around a vertex. (4) Last but not least, we design an Iterative Optimization Registration scheme (IOR) to calibrate the global axes of surfaces continuously and in turn adjust the vertices' signatures. This procedure effectively alleviates the sensitivity of HVS to the major axes of the objects being matched that may be ambiguous across individuals.

The rest of the paper is organized as follows. We present the definition of the Hierarchical Vertex Signature (HVS) and describe the Iterative Optimization Registration (IOR) scheme in section 2. Experimental results in a liver database are shown in section 3 , and we conclude the paper in section 4 .

\section{Method}

\subsection{Hierarchical Vertex Signature (HVS) in Canonical Coordinate Frame}

We treat the coordinates of the points on a 3D shape as random variables. Three principal components (PCs) $u_{1}$ to $u_{3}$ are calculated from their covariance matrix $C$. They are used to build up a canonical PCA coordinate frame. Suppose there are $N$ vertices on the surface, for each vertex $v_{i}, N-1$ spoke vectors $\overrightarrow{v_{i} v_{j}}$ are derived. A Relative Angle $(R A) \theta_{j}\left(v_{i}\right)$ is defined between the $j t h$ spoke vector of $v_{i}$ and the first principal axis. Two base-planes $\Pi_{12}\left(e_{2}^{T} \cdot v_{i}=0\right)$ and $\Pi_{13}\left(e_{3}^{T} \cdot v_{i}=0\right)$ are formed, where $e_{2}$ and $e_{3}$ are the second and the third eigenvectors of $C$ respectively. They are employed as reference planes to extend $\theta_{j}\left(v_{i}\right)$ from $[0, \pi]$ to $[-\pi, \pi]$. Furthermore, $R A$ s are normalized to $0 \sim 2 \pi$. For sake of paper space, please refer to our previous paper [14] to get the accurate formulation of $\theta_{j}\left(v_{i}\right)$.

Now what we want is investigating the distribution of these $R A$ s in the different fields of the vertex's neighborhood. The neighborhood relationship can be constructed from the triangular meshes of surfaces. There are many standard algorithms to build triangular meshes for an unorganized point-set such as [13]. Actually, the strict definition and exact connectivity of the meshes are not necessary for our method. Please refer to [14] for the neighborhood construction. After obtain the neighborhood map of the point-set, the distributions of the relative angles in different fields of neighborhood are concatenated to form a Hierarchical Vertex Signature (HVS) for a given surface point (ordered from near neighborhood fields to distant neighborhood fields). Obviously, in this way, each HVS contains hierarchical shape description around a vexel in a concise form. Since the $R A$ s are calculated with respect to the major axes of the organ, it captures the topological information of a point on a surface. We treat HVSs as sufficient and salient shape descriptors to register vertices between tissue surfaces. In practice, stochastic methods are employed to evaluate samples of HVSs, and histograms are constructed by counting how many samples fall into certain sized bins. Relative angles are rounded depended on the number of the histogram bins. Promisingly, HVS has the following properties: 
(1) Invariance: One of the basic characteristics of angles is that they are invariant to scale and rigid motions. In addition, since the $R A$ s are defined in the global canonical reference frame, they are translation and rotation invariant. Therefore, HVS can be directly employed for point registration without normalization.

(2) Robustness: As a bonus of stochastic methods, HVS is insensitive to noise, unsmoothness, small perturbations and points missing of organ surface, which are inevitably existed in 3D biomedical volumes recorded by different modalities.

(3) Efficiency: After obtaining the neighborhood map of a point-set, the complexity of HVS extraction is $O\left(N^{2}\right)$. The neighborhood map can be established and stored in advance prior to real-time registration.

(4) Hierarchy: HVS is a piecewise curve concatenated from the distributions in different neighborhood fields of a point. It expresses the local features of a surface when the layer of field is small, and represents the global shape when the layer becomes large. The last piece of HVS (See Fig.3) represents the distribution of the overall surface and provides a detailed and compact description of the global shape context. Therefore, we can claim that HVS captures both local and global spatial information around a given vertex in a hierarchical paradigm.

\subsection{Iterative Optimization 3D Point-Set Registration (IOP) Based on HVSs}

Our hypothesis is that HVSs of the correspondence pairs on tissue surfaces should be similar so the best matching distributions lead us to the most suitable point for registration. That is, we postulate that the HVSs associated with a pair of corresponding points possess the same theoretical form, even though they may be subjected to some forms of distortion and noise. As HVSs are represented as bins, it is natural to use Goodness-Of-Fit $\chi^{2}$ test to measure the dissimilarity factor $d f$ between points:

$$
d f\left(v_{i}, v_{j}^{\prime}\right)=\sum_{k=0}^{2 \pi} \frac{\left[\left(p_{k}\left(v_{i}\right) * S_{l}\left(v_{i}\right)-p_{k}^{\prime}\left(v_{j}^{\prime}\right) * S_{l}^{\prime}\left(v_{j}^{\prime}\right)\right]^{2}\right.}{p_{k}\left(v_{i}\right) * S_{l}\left(v_{l}\right)}
$$

where $v$ and $v^{\prime}$ are points in sample and target point-sets respectively. $p_{k}$ is probability value of the $k$ th bin, and $S_{l}$ is the vertex number in the $l$ th field. The range of $d f$ is $[0,1]$, and the best registration result is achieved by searching for a point on the target surface which has the minimum $d f$ with a given point on the sample surface.

We observe that very close vertices in dense point-sets may have similar global $R A$ distributions, but they have large differences between local structures (see the first piece of the distribution curve in Fig. 4). However, local distributions collected in the small neighborhood fields may contain much uncertainty and noise so they are not as robust as the global ones. Intuitively, HVSs of coarse point-set should be more distinctive since the vertices are sparse and distant so the spoke vectors of neighbors may not have similar orientations. Here, we propose an Iterative Optimization Registration scheme (IOR) for multi-resolution point-sets to perform accurate and robust registration in a hierarchical manner. The flowchart of IOS is elaborated in Fig. 1. 


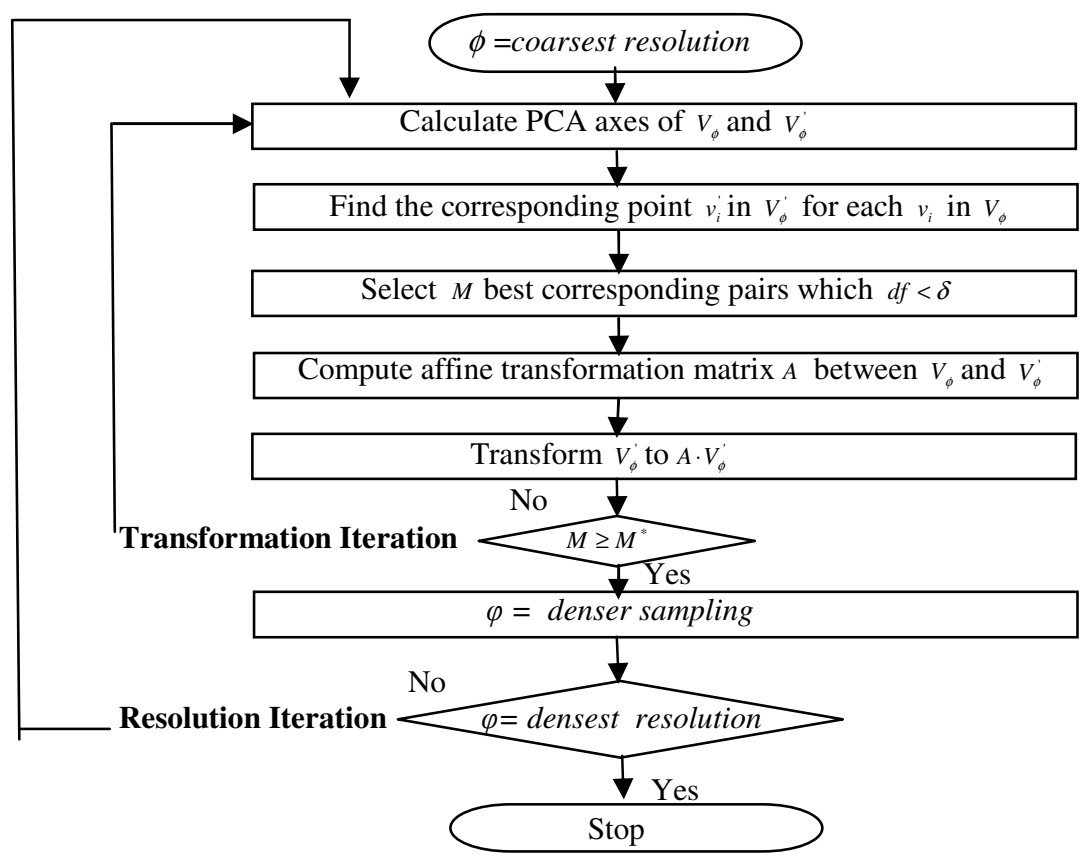

Fig. 1. The flowchart of the iterative optimization scheme for 3D point registration

Our approach consists of two main nested iterative schemes: resolution iteration and transformation iteration. During the resolution iteration, the registration begins with the coarsest point-sets ${ }^{1}$ and the correspondence searching is conducted in the range of the whole surface. After finding $M^{*}$ corresponding pairs and obtaining a rough alignment between surfaces by affine transformation, registration moves to denser sampling (higher resolution) data. At this stage, since the basic correspondence is established, the search range for a given vertex is limited to a small region. As we have mentioned, $R A$ s are calculated relative to the global coordinate frame, slight deformation of shapes may change orientations of major axes and accordingly influence the distributions of surface points. To achieve robust registration, a transformation iteration is designed specifically to correct and adjust the PCA axes continuously for the target point-set based on its affine transformation (AI). The consistency of major axes improves significantly the matching of the corresponding HVSs match (See Fig. 2). In the transformation iteration, at least $10 \%$ of the best corresponding pairs are selected which are approximately evenly distributed over the surface. The affine transformation matrix is calculated in a minimum error sense by:

$$
A=\left(V \cdot V^{\prime T}\right)\left(V^{\prime} \cdot V^{\prime T}\right)^{-1}
$$

\footnotetext{
${ }^{1}$ Please note that too much sub-sampling may make the blobby surface hard to find rotations.
} 


\section{Experiments and Results}

We have implemented IOR using C++ on PCs (1.8 G CPU, 1 G memory), and tested it in a liver image database. Each liver volume is defined by 20-30 CT slices. Livers are roundish and approximately symmetric soft tissues which frequently cause other registration techniques to fail. Imaging and analysis of the livers are also challenging due to potentially significant deformations among individuals and the lack of well-defined boundaries in the associated CT images. The shapes of the livers used in this work are semi-automatically segmented from the CT volumes. It has taken 5 minutes to generate the neighborhood map for the densest point-sets (1474 points). HVS extraction and point registration between two surfaces have taken less than 1 minute. The transform iteration usually converged after $3 \sim 5$ iterations. Three different resolution point-sets $(98,370$ and 1474) have been used for the evaluation by evenly sub-sampling the dense surfaces points. Fig. 2 shows an example of the alignment process between the PCA axes of the aligning livers during the lowest resolution point-set registration. The accuracy rates of the established point correspondence between the 12 livers used in the experiment and a sample are plotted in Fig. 2(d). The ground truth used here has been established in advanced by radiography specialists.
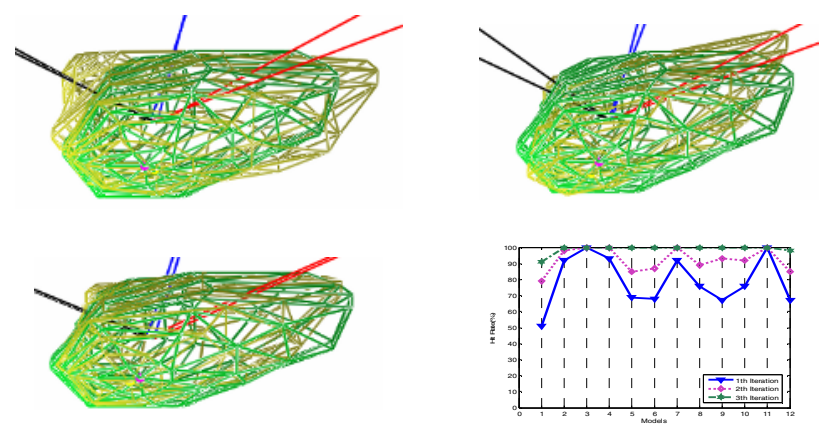

Fig. 2. (a-c) Alignment between PCA axes and affine transformation between the sample (yellow meshes) and the target surfaces (green meshes), red, blue and black axes are $u_{1}, u_{2}$ and $u_{3}$. (d) Accuracy rates comparison of point correspondence in different transformation iteration.

To demonstrate the highly discriminate ability of HVSs, in figure 3 , we show the established point correspondences between a few selected vertices on the liver surfaces. For better visualization, the surfaces are rendered by standard method. The HVSs of point $A$ and its registration partner $A^{\prime}$ are plotted in Fig. 3(b), 5 neighborhood fields and 72 bins are used. It is not difficult to discover that the resulting registration is visually correct, and their profiles of the HVSs match rather well. To verify that the distributions of corresponding points indeed follow the same theoretical function, we overlap the HVSs of the registration partners of point B on 14 liver surfaces, using 3 neighbor fields and 72 bins (Fig. 3c). The shapes of the curves are highly consistent. 

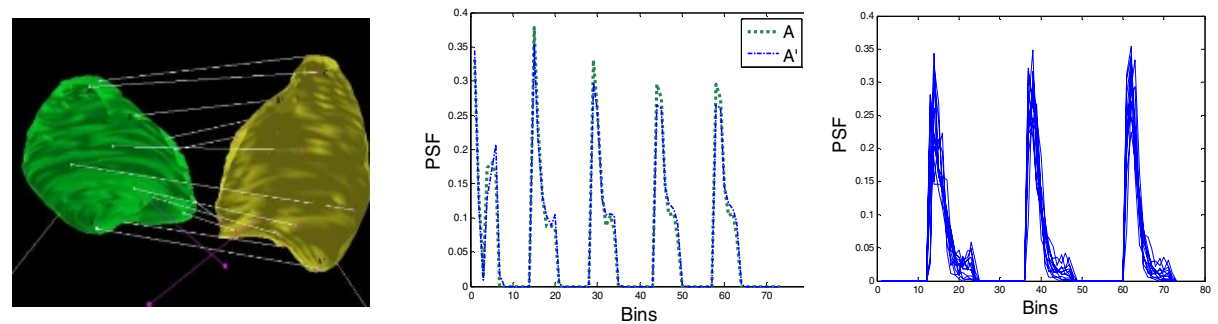

Fig. 3. (a) Point-set registration result (b) Overlapping HVSs of A and A'. (c) Overlapped HVSs of 14 corresponding points of $\mathrm{B}$.
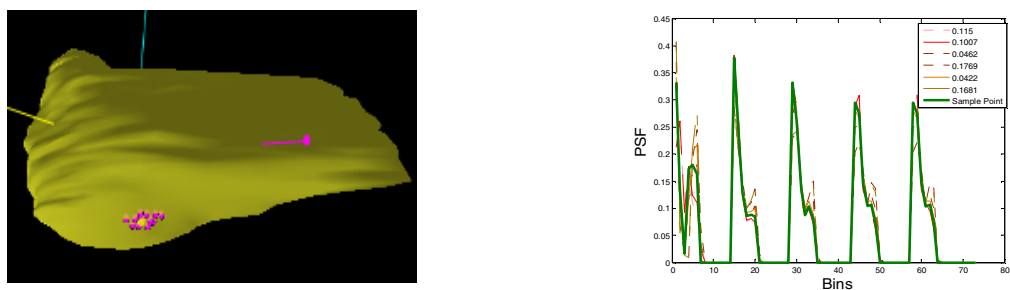

Fig. 4. (a) 12 vertices (pink dots) which have the lowest $d f$ with a sample vertex (yellow dot) (b) overlapping HVS curves of these vertices (Green curve is the distribution of sample vertex).

In Fig. 4a, we mark 12 vertices that have the lowest $d f$ value with a sample vertex. We can find that the vertices which have the most similar HVS distributions converge near the neighborhood of the sample vertex. The overlapping HVSs of these 12 vertices are plotted in Fig. 4b. The plots show high consistency in the global shapes (the last pieces of curves) but have large discrepancies in local structures, especially in the first neighbor field (the first piece of curves).

\section{Conclusion}

It is a fundamental yet still an open problem in computer vision to match two point-sets. In this paper, we propose a 3D point-set registration scheme for blobby biomedical objects. The angles $(R A)$ of the spoke vectors derived from a vertex relative to PCA axes are defined and the concatenated distributions of the RAs in the different neighborhood fields around a vertex are used to describe a Hierarchical Vertex Signature (HVS). HVS has many good characteristics such as invariance, robustness and hierarchy. It is also computation efficient. We have also proposed an iterative optimization registration scheme (IOR) in which the directions of major axes if the surfaces are calibrated incrementally according to affine transformation alignment between multi-resolution point-sets. The experiments indicate that our algorithm produces robust registration results for deformable organs such as the liver. 
The proposed techniques of registration and the establishment of 3D point correspondences are applicable to many other applications such as morphological or pathological analysis, 3D model retrieval and 3D object recognition.

\section{References}

1. Thirion, J.P., "Extremeal Points: Definition and Application to 3D image Registration", Proceedings CVPR '94, Pages: 587-592.

2. A. Liu, E. Bullitt, S. M. Pizer, "3D/2D Registration via Skeletal near Projective Invariance in Tubular Objects", Lecture Notes in Computer Science 1496: MICCAI 98, Springer, New York, 1998, Pages: 952-963.

3. T.F.Cootes, C.J.Taylor, D.H.Cooper and J.Graham et.al, "Active Shape Models-Their Training and Applications",CVIU Vol. 61 No. 1, 1995, Pages: 38-59.

4. P.J. Besl and N.D. McKay, "A Method for Registration of 3-D Shapes", PAMI Vol. 14(2), 1992, Pages: 239-256.

5. Cyr, C.M. Kamal, A.F. Sebastian, T.B. Kimia, B.B., "2D-3D registration based on shape matching", Proceedings of IEEE Workshop on Mathematical Methods in Biomedical Image Analysis, 2000, Pages: 198 - 203.

6. Sundar, H.; Silver, D.; Gagvani, N.; Dickinson, S, "Skeleton based shape matching and retrieval", Shape Modeling International, 2003, Pages:130 - 139.

7. Stan Scalroff et.al, "Modal Matching for Correspondence and Recognition", PAMI Vol. 17, No. 6, 1995, Pages: 545-561.

8. Marco Carcassoni et.al, "Correspondence Matching with Modal Clusters", PAMI Vol. 25, No. 12, 2003, Pages: 1609-1615.

9. Ehud Rivilin and Issac Weiss, "Local Invariants For Recognition”, PAMI Vol. 17 No. 3, 1995, Pages: 226-238.

10. Belongie, S. Malik, J. Puzicha, J., "Shape matching and object recognition using shape contexts", PAMI, Vol. 24 No. 4, 2002, Pages: 509-522.

11. Yamany, S.M. Farag, A.A., "Surface signatures: an orientation independent free-form surface representation scheme for the purpose of objects registration and matching", PAMI Vol. 24 No. 8, 2002, Pages: 1105-1120.

12. Chin Seng Chua et.al, "Point Signatures: A New Representation for 3D Object Recognition", IJCV Vol. 25 No. 1, 1997, Pages: 63-85.

13. Hoppe, H. DeRose, T.,Duchamp, T. ScDonald, J and Stuetzle, W., "Surface Reconstruction from Unorganised Points", Computer Graphics, 26(2), 1992, Pages: 71-78.

14. Jun Feng, Horace H. S. Ip, "Chi-Square Goodness-of-Fit Test of 3D Point Correspondence for Model Similarity Measure and Analysis", Lecture Notes in Computer Science, CIVR 2005, LNCS 3568, Pages: 445-453. 